\title{
Improving cable length measurements for large CDPR using the Vernier principle
}

\author{
Jean-Pierre Merlet \\ J-P. Merlet, HEPHAISTOS project, Université Côte d'Azur, Inria, France \\ Jean-Pierre.Merlet@inria.fr
}

\begin{abstract}
Cable lengths is an important input for determining the state of a CDPR. Using drum with helical guide may be appropriate for small or mediumsized CDPR but are problematic for large one. Another issue is the initialization of the cable length as measurements based on drum rotation are incremental. We propose to address automatic initialization and improvement of cable length measurements by using regularly spaced color marks on the cable combined with color sensors in the mast of the CDPR. We show that this disposition allows one to automate the initialization issue and then how it allows to get regularly accurate estimation of the cable length by using the Vernier principle.
\end{abstract}

Keywords: cable length, accuracy, initialization, calibration

\section{Introduction}

Measuring the cable lengths of CDPR with rotary winch is usually done by measuring the rotation of the drum with an encoder. Coiling the cable on the drum is usually done in two manners: the drum may have a spiral guide and a guiding mechanism moves synchronously the cable in front of the free part of the spiral or the cable is just coiled on the fly on the drum. An alternate to using rotary actuators is the use of linear actuator with a pulley mechanism that amplifies the stroke of the actuator [Merlet(2008)] but we will not consider this case in this paper. The spiral-guided mechanism is the one used in many cases for small to medium sized CDPR such as IPANEMA [Pott et al(2012)] or COGIRO [Gouttefarde et al(2012)]. It leads to a one-to-one and fixed relationship between the cable length and the drum rotation and if elasticity can be neglected it provides an accurate measurement of the cable length but it has drawbacks:

- the drum may accept only a single layer. This limits the available total cable length as increasing the drum radius will both increases the length measurement inaccuracy due to error in the drum rotation measurement and the necessary motor torque for a given cable tension. Hence such a mechanism may not be appropriate for very large CDPR that involves coiling several dizains or hundreds meters of cable

- the friction of the cable on the spiral guide increases the cable wear and cable elasticity is not taken into account

- according to the cable tension the cable may jump to another part of the guide 
Another issue for such a winch system is that the drum encoder usually provides only a relative measurement. When starting the CDPR it is therefore necessary either to calibrate the platform's pose [Alexandre(2012)], [Alexandre et al(2013)], [Chen(2004)], [Baczynski(2010)], [Miermeister and Pott(2012)] or to measure the initial length of the cable, both tasks being tedious. To the best of the author knowledge an automatic determination of the initial cable lengths has not yet been presented. We propose a method that allows both to obtain the initial cable lengths and provides regularly information on the cable length.

\section{Approach}

Our method is directly inspired by a method we have implemented successfully on our MARIONET-ASSIST CDPR [Merlet(2010)]. This CDPR uses synthetic cables and we have glued several aluminium foils on the cables at known distance from the platform cable attachment point $B$. At the winch level the cable goes through two electrically isolated Delrin guides that have been covered with aluminium foils, the mid-point between the guides being the output point $A$ of the winch. Each time a cable foil goes through the guides an electric contact is established providing a boolean information for the control computer. Using this event a semi-automated procedure may be used for initialization (the robot stops when detecting a foil and the operator input manually the corresponding cable length) and, in operation, for updating the current estimation of the cable length. In this paper we propose to improve this method by using colored marks on the cable and several color sensors in the mast that constitute the support structure of the CDPR (figure 1) for benefiting from a Vernier scale. Color sensor consists in leds that provides a constant illumination and receptors that are sensitive to a particular color (figure 1). Such sensors are inexpensive and our tests have shown that they can reliably detect at least the three RGB colors. They can easily be integrated in the support mast in small non transparent boxes with a circular opening for the cable (figure 1), the color sensor being protected from external illumination.


Fig. 1. A color sensor and the principle of color marks on the cable and color sensor in the mast 


\section{The initialization problem: a first approach}

A major problem for CDPR is to determine automatically what are the cable lengths at the start of the operation. The basic idea of our approach for automatizing this initialization process is the detection by a color sensor of $n$ successive color marks when coiling (or uncoiling) the cable, while having disposed the colors marks on the cable in such a way that there is only a single occurrence of any $n$ successive colors on the cable. For example if we use 3 possible colors R, G, B for the mark, then there will be, for example, a single GBR sequence on the whole cable, the other sequences being any triplet among the set (R, B, G), possibly with repetition (for example GGG or GGB). Let us assume that a given color sequence $\left(n_{1}, n_{2}, n_{3}\right)$ has been detected and that $d_{l}$ is the known distance between $A$ and the color sensor. As a given color sequence $\left(n_{1}, n_{2}, n_{3}\right)$ is unique on the cable the detection of $n_{3}$ gives us the corresponding mark number on the cable and consequently the distance $d$ between $B$ and the mark. The cable length $\rho=\|\mathbf{A B}\|$ is therefore obtained as $\rho=d-d_{l}$. Hence there is a one-to-one relationship between all possible sequences $\left(n_{1}, n_{2}, n_{3}\right)$ and $\rho$. Therefore the initialization method consists simply in uncoiling the cable until we have at least $n$ marks between a color sensor and the $B$ point and then coiling the cable until the color sensor has detected $n$ marks, such a process being easy to automatize. A faster initialization process will be presented in section 5.3.

\section{Number of marks}

As will be presented in the next sections we also intend to use the marks to update the current cable length. Intuitively it makes sense to have a large number of marks on the cable for that purpose, but our initialization process imposes the constraint of having a single occurrence of $n$ successive marks, whatever is the color combination. We will now investigate the influence of this constraint on the maximal number of marks on a cable. Assume that we use marks with $k$ different colors: our problem is to determine the maximal number of marks $m$ that we may have on the cable so that all subsets of $n$ successive marks are different in term of color value. In other words we have to determine the largest color sequence that satisfy this constraint. This problem is well known in combinatorial theory and such a sequence is called a De Bruijn sequence [Bruijn(1975),Bruijn(1946)]. For a cable with $k$ possible mark values that has all $n$ combination of the mark values a single time in the sequence, the sequence length (i.e. the number of marks) is $k^{n}$ for a cyclic sequence. So for $n=k=3$ we have a sequence of length 27. But as our sequence is not cyclic we may add 2 additional marks (but not 3 , which will be contradictory) so we may have up to 29 marks on the cable. An example of such sequence (the colors are indicated by the number 1, 2, 3) is: [ 12312112213222323313111333212 ] . Using this coding the detection of 3 successive colors by a color sensor allows one to determine the location of the last mark on the cable and consequently the cable length.

For $n=4$ the De Bruijn sequence has a length of $3^{4}=81$. and we may add 3 additional marks while keeping the property so that we will end up with a total of 84 marks on the cable. For $k=4$ colors and a sequence of $n=3$ marks the De Bruijn 
sequence has a length of 64 to which we may add 2 marks for a total of 66 marks while for a sequence of $n=4$ marks we will have a total of 259 marks on the cables. We have now to examine how the marks may be used to determine the cable length.

\section{Measuring cable lengths}

The height of the tower will be denoted $h$ (in the examples we will assume $h=15$ ) and we assume that there is a color sensor every $d_{m}$ meters starting from the base of the tower so that we have $k_{\max }$ sensors in the tower with $k_{\max }=$ floor $\left(h / d_{m}\right)$, where floor is the largest integer lower than $h / d_{m}$. The cable sensor will be numbered from 1 to $k_{\max }$, the sensor $k_{\max }$ being the highest in the tower. We have $k_{\max }^{1}$ marks on the cable that are assumed to be distributed regularly on the cable, the distance between two successive marks being denoted by $d_{c}$ meters. The marks are numbered from 1 to $k_{\text {max }}^{1}$, mark $k_{\text {max }}^{1}$ being the one the closest to $B$. The distance between mark $k_{\max }^{1}$ and the attachment point $B$, called the dead length, will be denoted by $b$. When the cable is completely uncoiled we assume that the first mark on the cable is located at the $k_{\text {max }}$ sensor. The total length $L_{t}$ of the cable between the base of the tower and $B$ is

$$
L_{t}=k_{\max } d_{m}+\left(k_{\max }^{1}-1\right) d_{c}+b
$$

If we assume that the $k_{1}$-th mark is located at the $k$-th color sensor, then the cable length $L$ between the color sensor and $B$ is:

$$
L=\left(k_{\max }^{1}-k_{1}\right) d_{c}+b
$$

and the length $\rho$ of the cable between $A, B$ is then obtained from $L$ by subtracting the distance $h-k d_{m}$ between the $k$-th sensor and the top of the tower:

$$
\rho=\left(k_{\max }^{1}-k_{1}\right) d_{c}+b-\left(h-k d_{m}\right)
$$

Consequently the lowest value $\rho_{\min }$ for $\rho$ is obtained for $k_{1}=k_{\max }^{1}$ and $k=1$ with the value $\rho_{\min }=b-h+d_{m}$

We will choose $b=h-d_{m}$ so that this minimum is 0 in order to have always positive $\rho$ when a mark lies in front of a color sensor. Consequently $\rho$ is obtained as:

$$
\rho=\left(k_{\max }^{1}-k_{1}\right) d_{c}+(k-1) d_{m}
$$

By taking all possible values for $k, k_{1}$ we get all $\rho$ that will correspond to a mark detection by a color sensor, that we will call a sensor event, and is defined by a triplet $\left(k_{1}, k, \rho\right)$ corresponding respectively to the mark number, to the sensor number and the corresponding cable length.. As $k, k_{1}$ lie respectively in $\left[1, k_{\max }\right],\left[1, k_{\max }^{1}\right]$ there will be at most $k_{\max }^{1} k_{\max }$ different $\rho$. This is an upper bound as the same $\rho$ may possibly be obtained for different pairs $\left(k_{1}, k\right)$. For these values of $\rho$ we will get a sensor event that will allow us to determine the cable length between $A, B$.

Sorting by increasing value all possible values of $\rho$ and taking the differences between successive values will provide the various cable length changes $\Delta \rho$ between two sensor events. Note that $\Delta \rho$ cannot exceed $d_{m}$ which correspond to the case where a 
given mark is seen successively by the same color sensor. Between two sensor events the cable length will be interpolated from the measurement of the drum rotation with some uncertainty because of modeling error on the coiling process. Clearly there is an interest of having the largest possible number of significant sensor event (i.e. one that provides an update on the cable length), a relatively flat distribution of the $\Delta \rho$ with a low average value. Another interest of a flat distribution is that we may relate two successive sensor events (which will provide a change $\Delta \rho$ in the cable length) to the corresponding drum rotation $\Delta \theta$ in order to obtain an estimate of the mean drum radius that will be updated at each sensor event. This estimate will then be used to determine the cable length between two sensor events.

\subsection{Significant sensor events}

Clearly we are interested in having the largest number of different $\rho$. Consequently we should avoid having two sensor events for the same $\rho$. In other words we have to determine if we may have $\Delta \rho$ equal to 0 being given $d_{m}, d_{c}$. Consider now 2 sensor events defined by the triplets $\left(k_{1}, k, \rho\right),\left(k_{1}^{\prime}, k^{\prime}, \rho^{\prime}\right)$. Using equation (4) we may calculate the $\Delta \rho=\left|\rho-\rho^{\prime}\right|$ as

$$
\Delta \rho=\left|\left(k_{1}^{\prime}-k_{1}\right) d_{c}+\left(k-k^{\prime}\right) d_{m}\right|
$$

There is a symmetry in this relation as, being given the events $\left(k_{1}, k\right),\left(k_{1}^{\prime}, k^{\prime}\right)$ we will get the same $\Delta \rho$ for the events $\left(k_{1}^{\prime}-k_{\max }^{1}, k_{\max }-k^{\prime}\right),\left(k_{\max }^{1}-k_{1}, k_{\max }-k^{\prime}\right)$. Let us assume that the ratio $d_{m} / d_{c}$ is a rational number $p / q$ and consider the rational number

$$
\frac{p_{1}}{q_{1}}=\frac{k_{1}-k_{1}^{\prime}}{\left(k-k^{\prime}\right)}
$$

which is such that $\left|p_{1}\right|$ is the lowest possible value for $\left|k_{1}-k_{1}^{\prime}\right|$ while $\left|q_{1}\right|$ is the minimal value of $\left|k-k^{\prime}\right|$. We have

$$
\Delta \rho=d_{c}\left|\left(k-k^{\prime}\right)\left(\frac{-p_{1}}{q_{1}}+\frac{p}{q}\right)\right|
$$

As $d_{c}$ is a known positive constant the minimum of $\Delta \rho$ will be obtained when $\mid(k-$ $\left.k^{\prime}\right)\left(\frac{-p_{1}}{q_{1}}+\frac{p}{q}\right) \mid$ is minimal. As $\left|\left(k-k^{\prime}\right)\right| \geq\left|q_{1}\right|$ the minimum of $\Delta \rho$ is obtained for the rational $p_{1} / q_{1}$ that is the closest to $p / q$.

Equation (5) is essential to assert the distribution of the $\Delta \rho$. For example for $k_{\text {max }}^{1}=$ 29 (29 marks on the cable) and $d_{m}=2.4$ (which leads to $k_{\max }=6$ ) and $d_{c}=2$ we get $d_{m} / d_{c}=1.2=6 / 5$. If we set $p_{1}=6, q_{1}=5$ we get $k_{1}-k_{1}^{\prime}=6$ leading to $k_{1}=$ $k_{1}^{\prime}+6 \in[7,29]$ and $k_{1}^{\prime} \in[1,23]$. We have also $k-k^{\prime}=5$ and as $k$ cannot exceed 6 we get $k^{\prime}=1, k=6$. The $\rho$ obtained for the pair $\left(k_{1} \in[7,29], 6\right)$ will be the same than for the pair $\left(k_{1}-6,1\right)$ so that we will get 23 identical $\rho$. As the maximum number of $\rho$ is $29 \times 6=174$ there will be $174-23=151$ different $\rho$ available. .

However we are not only interested in discarding the sensor events that will give the same $\rho$ but also in the set of $\Delta \rho$ that are lower than $d_{m}$. Hence we have to find the positive rationals $p_{1} / q_{1}$ with a denominator at most equal to $k_{\max }-1$ and lower or equal to $k_{\text {max }}^{1}$ that will lead to $|\Delta \rho|$ that are lower of equal to $d_{m}$. For that purpose we consider 
the following theorem that is used for studying the Farey sequences ${ }^{1}$ :

Theorem 1: Let $s=a / b$ and $t=c / d>s$ and if there is no rational between $s$ and $t$ with a denominator that is lower than the largest $b$ or $d$, then $b c-a d=1$ and the rational with smallest denominator between $s$ and $t$ is $\frac{a+c}{b+d}$.

Let us illustrate the application of this theorem in our previous example for $d_{m}=2.4$ and $d_{c}=2$. We have $t=p / q=6 / 5$ and we are looking for $s$ that is smaller than $t$ so that there is no rational between $s, t$ with a denominator that is smaller than $a, 5$. According to the theorem we must have $6 b-5 a=1$ that is clearly satisfied for $a=$ $b=1$. Then according to the theorem the next rationals having the lowest denominator are $7 / 6,13 / 11$ and so on with increasing denominator. As the denominator are larger than 5 , then the closest valid rational $p_{1} / q_{1}$ to $p / q$, that is lower than $p / q$, is $1 / 1$. As $b=k-k^{\prime}$ we get the minimal $\Delta \rho$ as $d_{c} \times(-1+6 / 5)=d_{c} / 5=0.4$. For any $\rho$ obtained for the pair $\left(k_{1}, k\right)$, then the $\rho$ obtained for the pair $\left(k_{1}+1, k+1\right)$ will differ from the previous one by 0.4 .

We may now examine the closest valid rational $p_{1} / q_{1}$ to $p / q$, that is larger than $p / q$. Using the theorem we get the condition $5 c-6 d=1$ that is fulfilled by $c=5, d=4$ leading also to $\Delta \rho=0.4$. For being more systematic we may use the following theorem

Theorem 2: let two rationals $a / b$ and $c / d$ and let $u=p / q$ the rational closest to $c / d$ and larger than $c / d$ with denominator lower or equal to $n$. Let $k$ be the largest integer such that $k \leq(n+b) / d$. The value of $p, q$ are given by

$$
p=k c-a \quad q=k d-b
$$

We will use this theorem by starting from the lowest possible successive value for $a / b, c / d$ which are $1 /\left(k_{\max }-1\right), 1 /\left(k_{\max }-2\right)$ and construct the full Farey sequence or order 5 corresponding to $. d_{m}=2.4$ and $d_{c}=2$. We get the following pairs $(p / q,|\Delta \rho|)$ : $(1 / 1$ or $5 / 4,0.4),(4 / 3,0.8),(3 / 2,1.2),(2 / 1,1.6),(7 / 5,2)$. Note that $\Delta \rho=d_{m}$ is always possible by setting $k_{1}=1, k=k_{\max }, k=k_{\max }-1$. But although we have obtained what are the possible values of $\Delta \rho$ we have not established their frequency for a given configuration. The dead length is $b=12.6$ and measurements for $\rho$ between 2 and 68 will be obtained. We get 142 successive $\rho$ that differs by $0.4,2$ that differs by $0.8,1.2,1.6$ and 1 that differ by 2 . The $\Delta \rho$ distribution as a function of the cable length $\rho$ is provided in figure 2. As may be seen, apart at the extremity, the $\Delta \rho$ is everywhere equal to 0.4 with a mean value of 0.442 and a variance of 0.04571 .

Let us now consider that $d_{m}=1.3, d_{c}=2$ so that $d_{m} / d_{c}=13 / 20$ and $k_{\text {max }}=11$. We get the following pair $(p / q,|\Delta \rho|):(2 / 3,0.1),(5 / 8,0.4),(1 / 2,0.6),(1 / 1,0.7),(3 / 4$, $0.8),(5 / 7,0.9),(7 / 10,1),(4 / 7,1.1)$ and $\Delta \rho=0.1$ may always be obtained . This is confirmed by the calculation with 216 measurement differing by $0.1,72$ by $0.4,12$ by $0.5,12$ by $0.6,4$ by 0.7 and 1 for 1.3 . The value of $b$ is 13.7 and we get measurements for $\rho$ between 1.3 and 69 (figure 2). The mean value of the $\Delta \rho$ is 0.213 and its variance is 0.0322 . It may be observed that although we have almost doubled the number of

\footnotetext{
${ }^{1}$ A Farey sequence of order $\mathrm{n}$ are the irreducible rationals between 0 and 1 whose denominator is lower or equal to $n$
} 

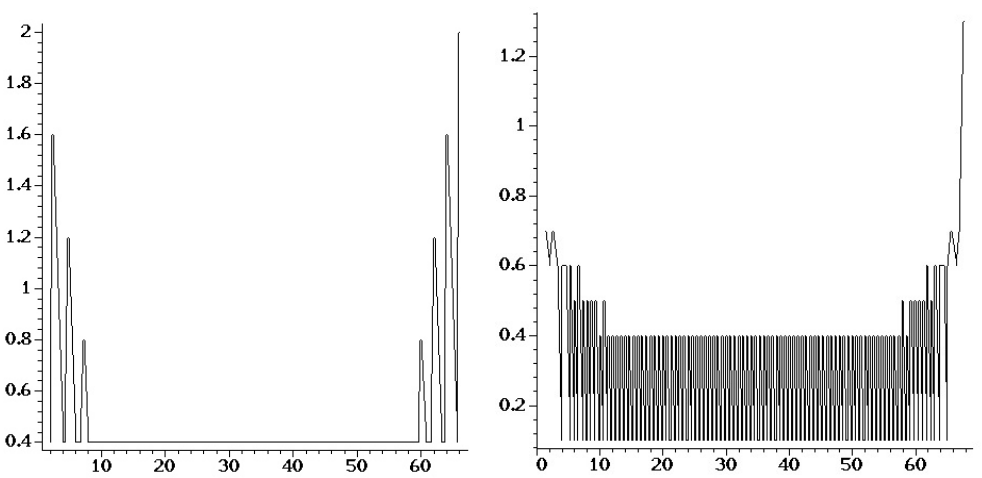

Fig. 2. On the left the distribution of $\Delta \rho$ as a function of $\rho$ for $d_{m}=2.4, d_{c}=2$ with 29 marks and 6 color sensors. On the right the distribution for $d_{m}=1.3, d_{c}=2$ with 29 marks and 11 color sensors.

color sensors compared to the previous case we still get a large number of cases with $\Delta \rho=0.4$ so that the choice of $d_{m}=1.3$ may not be optimal. Optimal configuration is addressed in the next section.

\subsection{Optimal configuration}

\section{Optimal choice of sensor distance $d_{m}$ for a given $d_{c}$}

A key point for realizing such a measurement system is first to determine what could be the number $k_{\max }$ of color sensors and then select the $d_{m}$ for the best performance. For a given $k_{\max }, d_{m}$ should lie in the range $\left.] h /\left(k_{\max }+1\right), h / k_{\max }\right]$. Consider for example that $h=15, k_{\max }=6, d_{c}=2$. We may draw the curve of the mean and variance values of the $\Delta \rho$ as a function of $d_{m}$, figure 3 . As may be seen the mean value of $\Delta \rho$ is an increasing function of $d_{m}$ while there is a discontinuity point for $d_{m}=2.4$ with a sudden increase of the mean value but also a sudden decrease of the variance.

To understand this behavior we have to consider how many minimal $\Delta \rho$ we will obtained for a given rational ratio $d_{m} / d_{c}=p / q$. For that purpose we have to look at the rational $p_{1} / q_{1}$ which has a denominator $q_{1}$ lower or equal to $k_{\max }$ and that is the closest to $p / q$. The larger the sets of possible $k_{1}, k$ are the larger will be the set of minimal $\Delta \rho$. As the values of $k_{1}, k$ are restricted to lie respectively in the range $\left[1, k_{\max }^{1}-\right.$ $\left.p_{1}\right],\left[1, k_{\max }-q_{1}\right]$ the larger the sets will be if $p_{1}, q_{1}$ are close to 1 .

Let us assume that $q$ is lower than $k_{\max }$. According to theorem 1 the closest rational $p_{1} / q_{1}$ that is lower than $p / q$ should satisfy $p p_{1}-q_{1} q=1$. If we set $p_{1}=q_{1}=1$ we get the constraint (A) $p-q=1$ so that $d_{m}=p d_{c} /(p-1)$ Hence $d_{m}$ is a decreasing function of $p$ that cannot be greater than $k_{\max }=6$. If we look at all possible value for $p$ between 1 and 6 , then we found out that (A) may be satisfied only for $p=6$. The corresponding $d_{m} / d_{c}$ ratio is $6 / 5=2.4$ and for this ratio we will get the maximal number of $\Delta \rho$ that are at the minimum (in this case $\Delta \rho=0.4$ as seen on figure 2). However for this value 

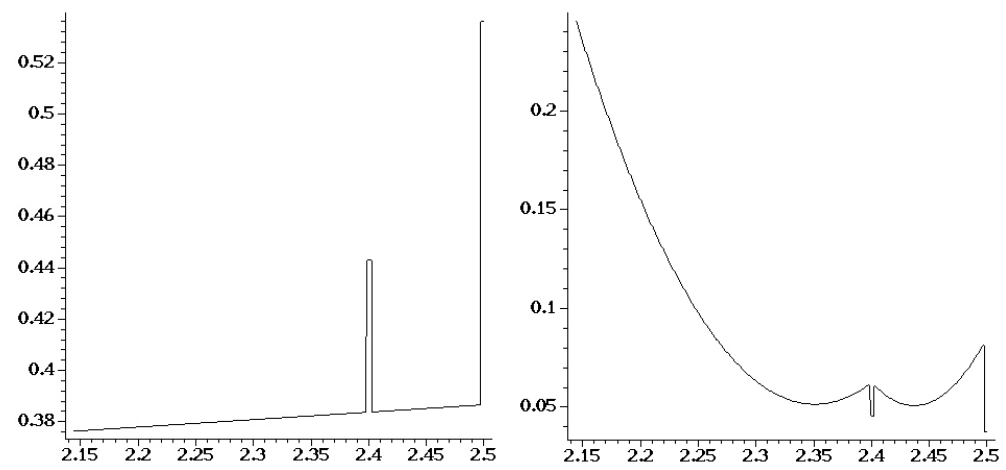

Fig. 3. The mean value and variance of $\Delta \rho$ as a function of the distance $d_{m}$ between the color sensor for $d_{c}=2$ and 29 color marks

the mean value is not that good: other value of $d_{m}$ may lead to a sequence of.$\Delta \rho$ whose minimum is lower than 0.4 .

Let us look now at $d_{m}=2.35$, a value that presents the second minimal value for the variance. For this value we get $23 \Delta \rho=0.25,140 \Delta \rho=0.35,2 \Delta \rho=0.6,0.95$, $1.3,1.65$ and $1 \Delta \rho=2$. Here we have a larger number of $\Delta \rho$ equal to 0.25 or 0.35 than compared to the value 0.4 obtained for $d_{m}=2.4$. Hence apparently for a given $d_{c}$ we shall consider as possible optimal values of $d_{m}$ the one having the lowest variances.

We may also consider increasing $d_{c}$ to a larger value for CDPR having large cable length. For example if we have 29 marks with $d_{c}=5, d_{m}=2.2$ (6 sensors) we get a measuring range of 151 meters with the following pairs of $\Delta \rho$ and their number: (0.6, $112),(1,27),(1.6,30)$. If we move to $d_{c}=10$ and the same number of sensors and $d_{m}$, then the measuring range increases to 291 meters with $(1,28),(1.2,56),(2.2,88)$.

\section{Influence of the number of marks}

Although the number of sensors in a system is important, these sensors are inexpensive and require a low level of maintenance while the marks will require more attention. We may thus consider having less than the maximum number of marks imposed by the the initialization process, but this will impose to have larger $d_{c}$ in order to have a sufficient total cable length.

As seen in the previous section the ratio $d_{m} / d_{c}=p / q$ that has the maximum of lowest $\Delta \rho$ is such the closest rational with a denominator lower or equal to $q$ should be $p_{1} / q_{1}=1 / 1$. Using theorem 1 we get $p-q=1$ if $p_{1} / q_{1}$ is lower than $p / q$ so that $d_{m}=d_{c} p /(p-1)$ provided that $p-1$ is lower or equal to the number of sensors floor $\left(h / d_{m}\right)$. If $p_{1} / q_{1}$ is greater than $p / q$ we get $q-p=1$ so that $d_{m}=d_{c} p /(p+1)$ Therefore being given $d_{c}$ we are able to find all the valid $d_{m}$. 
Assume that we will use only 20 marks instead of 29 but set $d_{c}$ to 3 in order to still have a large total cable length. For $d_{c}=3$ table 1 provides some examples of $d_{m}$ leading to interesting set of $\Delta \rho$ with their distribution.

\begin{tabular}{|l|c|c|c|c|}
\hline$d_{m}$ & $\rho_{\max }$ & number of sensors & $\Delta \rho$ & number \\
\hline 4.5 & 66 & 3 & $1.5-3$ & 401 \\
\hline 4 & 65 & 3 & $1-2-3$ & 5521 \\
\hline 3.75 & 68.25 & 4 & $0.75-1.5-2.25-3$ & 73221 \\
\hline 2.5 & 69.5 & 6 & $0.5-1-1.5-2-2.5$ & 1112221 \\
\hline 2.4 & 69 & 6 & $0.6-1.2-1.8-2.4$ & 97221 \\
\hline 2.15 & 67.75 & 6 & $0.4-0.45-0.85$ & 345427 \\
\hline 1.9 & 68.4 & 7 & $0.3-0.5-0.8-1.1-1,9$ & 72342641 \\
\hline 1.1 & 70.2 & 13 & $0.1-0.2-0.3-0.5-0.8-1.1$ & 3285126663 \\
\hline
\end{tabular}

Table 1. Minimal $\Delta \rho$ measurement and their number for various $d_{m}$ being given $d_{c}=3$ and 20 marks on the cable

It may be seen that the best compromises for 3 sensors is $d_{m}=4$ with an increase of accuracy for 4 sensors with $d_{m}=3.75$, while $d_{m}=2.5$ is optimal for 6 sensors. Note that we have added a line with $d_{m}=2.15$ that correspond to the lowest variance beside the one leading to the maximal number of minimal $\Delta \rho$ as it offers interesting performances. As may be seen from this table increasing the number of sensors may have a large benefit on the measurement. For 13 sensors we get 243 changes for $\Delta \rho$ in the range $[0.1,0.3]$.

We may now consider going into the opposite direction by increasing the number of marks in order to increase the accuracy of the system, at the price of requiring the reading of 4 marks to initialize the cable length (although this argument will be invalidated if we use the initialization procedure described in section 5.3). Consider first that $d_{c}=1.5$ so that we have 40 marks. We consider the case where the variance is minimal and table 2 summarizes the result. As it seems difficult to position the sensor with an

\begin{tabular}{|l|c|c|c|c|}
\hline$d_{m}$ & $\rho_{\max }$ & number of sensors & $\Delta \rho$ & number \\
\hline 2.4 & 69.75 & 6 & $0.3-0.6-0.9-1.5$ & 1891241 \\
\hline 2.25 & 70.5 & 6 & $0.75-1.5$ & 891 \\
\hline 1.6875 & 70.3125 & 8 & $0.1875 \ldots$ & 305 \\
\hline 1.8 & 71.1 & 8 & $0.3-0.6-0.9-1.2-1.5$ & 2092221 \\
\hline 1.83 & 71.31 & 8 & $0.15-0.18-0.33 \ldots$ & 10214069 \\
\hline 1.66 & 71.78 & 9 & $0.16-0.22-\ldots$ & 31231 \\
\hline 1.35 & 72 & 11 & $0.15-\ldots$ & 392 \\
\hline 1.27 & 71.2 & 11 & $0.11-0.12-0.23-\ldots$ & 136175118 \\
\hline
\end{tabular}

Table 2. Minimal $\Delta \rho$ measurement and their number for various $d_{m}$ being given $d_{c}=1.5$ and 40 marks on the cable 
accuracy of half a centimeter, the optimal solution for $d_{m}$ seem to be $2.4,1.8,1.83,1.66$, 1.35. Table 3 summarizes good design solutions for cable length in the range of $60-70$ meters.

\begin{tabular}{|l|c|c|c|c|c|}
\hline number of marks & $d_{c}$ & $d_{m}$ & number of sensors & $\Delta \rho$ & number \\
\hline 12 & 5 & 3.75 & 4 & 1.25 & 43 \\
\hline 15 & 4 & 3 & 5 & 1 & 58 \\
\hline 20 & 3 & 1 & 3 & 1 & 55 \\
\hline 20 & 3 & 3.75 & 4 & 0.75 & 73 \\
\hline 20 & 3 & 2.5 & 6 & 0.5 & 111 \\
\hline 29 & 2 & 2.4 & 6 & 0.4 & 142 \\
\hline 29 & 2 & 2.35 & 6 & $0.25-0.35$ & $23-140$ \\
\hline \hline 40 & 1.5 & 2.4 & 6 & $0.3-0.6$ & $189-12$ \\
\hline 40 & 1.5 & 1.83 & 8 & $0.15-0.18-0.33$ & $102-140-69$ \\
\hline 40 & 1.5 & 1.66 & 9 & $0.16-0.22$ & $312-31$ \\
\hline 40 & 1.5 & 1.35 & 11 & 0.15 & 392 \\
\hline 60 & 1 & 2.4 & 6 & 0.3 & 289 \\
\hline 60 & 1 & 1.75 & 8 & 0.25 & 363 \\
\hline 60 & 1 & 1.3 & 11 & 0.10 .2 & 375272 \\
\hline 84 & 0.7 & 1 & 15 & 0.1 & 639 \\
\hline
\end{tabular}

Table 3. Best system arrangement for various $d_{c}$ (distance between marks) and number of sensors ( $d_{m}=$ distance between color sensors)

\subsection{The initialization problem: a second approach}

We have proposed in section 3 a first approach to determine the initial cable length based on the detection of $n$ successive marks by one sensor, the coding of the cable being such that there is a single occurrence of a given sequence of $n$ colors on the cable. For the initialization process we therefore need to coil the cable by $n d_{c}$. However we may get a faster initialization process by taking into account that we have several color sensors in the mast so that we may use all event detection event for performing the initialization process. Clearly we aim at determining the cable length by coiling the cable by less than $n d_{c}$.

If we have $n$ different colors on the cable and $m$ marks on the cable so that $m$ is maximal, then we will have roughly $m / n$ marks of the same color. For example for $n=3, m=29$ and the marking presented in section 3 we have 10 marks of color 1,2 and 9 marks of color 3 . Remind that if the color sensor $n_{s}$ detects mark $m_{s}$, then the cable length $\rho$ is given by

$$
\rho=n_{s} d_{m}-h+L_{0}-\left(m_{s}-1\right) d_{c}
$$

where $L_{0}$ is the total length of the cable. After starting the coiling there will be a first sensor detection event that provide $n_{s}$ while $m_{s}$ is not known. However the detected 
color provides a limited choice for $m_{s}$ (at most 10 in our example) and consequently a limited set $\left\{\rho_{1}, \ldots, \rho_{l}\right\}$ of possible $\rho$. For each of the $\rho_{i}$ we consider the set of all possible values of $\rho$ that are lower than $\rho_{i}$ obtained for all possible values of $n_{s}, m_{s}$ and we then sort this set by decreasing value of $\rho$. The first element of the ordered set provides what will be the next sensor detection event if the current value of the $\rho$ is $\rho_{i}$. Hence we get a list $\mathscr{L}$ of $\left(\rho_{i}, n_{s}^{i}, c^{i}\right)$ where $n_{s}^{i}$ is the sensor number and $c^{i}$ is the mark color for the next event. When a new sensor event occurs we check the coherence of the $n_{s}$ and the color with each $n_{s}^{i}, c^{i}$ : each element of $\mathscr{L}$ that is not coherent with the sensor number or color is removed from $\mathscr{L}$. This leads to a new list that contains all possible values of the current value of $\rho$ and prediction about the next sensor event and we repeat the process. As the coherence test allows one to reduce the size of $\mathscr{L}$, we shall end up with a list with a single element that is the current value of $\rho$.

For example we consider the case where we have 29 marks separated by 2 meters with 3 different colors and 6 color sensors separated by 1.6 meter. If the cable length is $\rho=21.5$ when starting the initialization process, then the first detection event is color 3 on sensor 4. Using color 3 we obtain the list $\mathscr{L}=\{(45.4,5,1),(29.4,5,2),(21.4,5,2)$, $(17.4,5,3),(15.4,5,1),(11.4,5,1),(3.4,5,3),(1.4,5,3)\}$. The second sensor event is color 2 for sensor 5 meaning that among the previous list only the value 21.4 and 29.4 are coherent, leading to $\mathscr{L}=\{(29,6,2),(21,1,2)\}$. As the next event has color 2 and sensor 1 we may discard 29 and we will have determined that $\rho=20.2$, after coiling only 1.3 meter of cable. More generally figures 4 shows how much cable should be coiled before getting the current $\rho$ value as a function of the initial value of $\rho$ and the number of detection events that are necessary to identify this value.
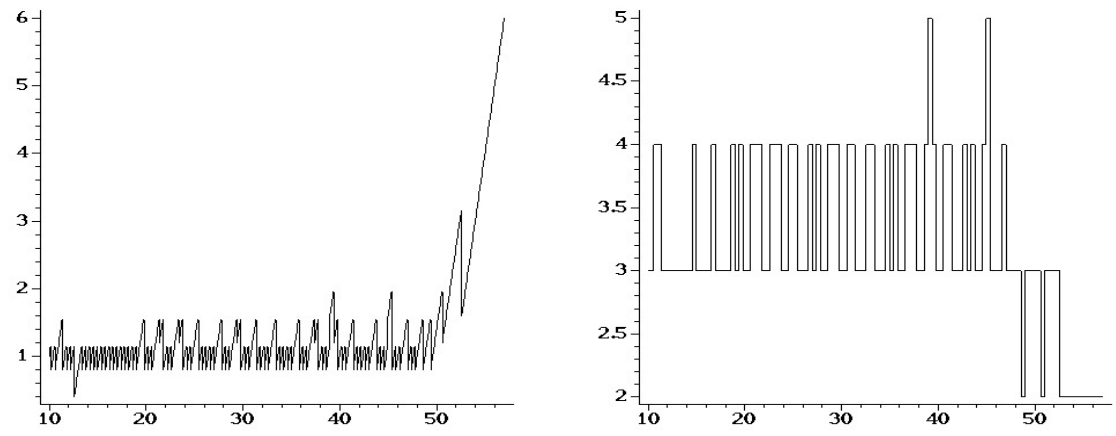

Fig. 4. On the left the coiling amount that is necessary to identify the current $\rho$ as a function of the $\rho$ value. On the right the number of sensor events that are necessary to identify the current $\rho$ as a function of the $\rho$ value ( 29 marks, 6 sensors, $d_{c}=1.6, d_{m}=2$ ).

As may be seen on these figures the average amount of coiling distance for the identification of the current $\rho$ is 1.069 meter (variance: 0.054 , min: 0.4 , max: 1.95 ) for an initial $\rho$ between 10 and 50 meter, much better than the value of 6 meters required 
for the method proposed in section 3. If we use $d_{m}=1.83, d_{c}=1.5,8$ color sensors and 40 marks, then in average we need only 0.622 meter of coiling (variance: 0.03 , min: 0.34, max: 1.29) for determining the current $\rho$.

\section{Conclusion}

In this paper we propose a setup for both allowing an automated determination of the initial cable lengths and improving the cable length measurements. The method is simple and allows one to obtain very good estimation of the current cable lengths especially for large CDPR (for example being able to provide the cable length for any change of 0.4 meter for a 60 meters cable). It allows also to get an estimate of the drum radius at each sensor event, thereby allowing to improve the estimation of the cable length between two sensor events. The method is robust: being given the sensor price it is possible to use sever color sensors within a given sensor box. Furthermore it is possible to detect sensor failure based on the absence of a color signal during a significant change of cable length. We intend also to explore if this system may not allow to estimate the cable elastic deformation by comparing the difference between the distance between marks (that is known at rest) and the distance observed between sensor events. On-line calibration may also benefit from being able to fix the cable lengths at known values.

\section{References}

[Baczynski(2010)] Baczynski J, Baczynski M (2010) Simple system for determining starting position of cable-driven manipulator. In: IEEE Int. Conf. on Computer Information Systems and Industrial Management Applications (CISIM), Cracow, pp 102-106

[Bruijn(1946)] Bruijn N (1946) A combinatorial problem. Indagationes Math 8:461-467

[Bruijn(1975)] Bruijn N (1975) Acknowledgment of priority to c. flye sainte-marie on the counting of circular arrangements of $2^{n}$ zeros and ones that show each n-letter word exactly once. Indhove: Technische Hogeschool Eidenhoven

[Chen(2004)] Chen Q, et al (2004) An integrated two-level self-calibration method for cabledriven manipulator. IEEE Trans on Robotics 10(2):380-391

[Gouttefarde et al(2012)] Gouttefarde M, et al (2012) Simplified static analysis of largedimension parallel cable-driven robots. In: IEEE Int. Conf. on Robotics and Automation, Saint Paul, pp 2299-2305

[Merlet(2008)] Merlet JP (2008) Kinematics of the wire-driven parallel robot MARIONET using linear actuators. In: IEEE Int. Conf. on Robotics and Automation, Pasadena

[Merlet(2010)] Merlet JP (2010) MARIONET, a family of modular wire-driven parallel robots. In: ARK, Piran, pp 53-62

[Miermeister and Pott(2012)] Miermeister P, Pott A (2012) Auto calibration method for cabledriven parallel robot using force sensors. In: ARK, Innsbruck, pp 269-276

[Pott et al(2012)] Pott A, et al (2012) IPAnema: a family of cable-driven parallel robots for industrial applications. In: 1st Int. Conf. on cable-driven parallel robots (CableCon), Stuttgart, pp 119-134

[Alexandre(2012)] Alexandre dit Sandretto J, Daney D, Gouttefarde M (2012) Calibration of a fully-constrained parallel cable-driven robot. In: RoManSy, Paris, pp 12-41

[Alexandre et al(2013)] Alexandre dit Sandretto J, et al (2013) Certified calibration of a cable-driven robot using interval contractor programming. In: Computational Kinematics, Barcelona 